\title{
Advanced separation technologies for pre-concentration of metal ores and the additional process control
}

\author{
Jacek Kolacz ${ }^{1, *}$ \\ ${ }^{1}$ Comex AS, Box 53, 1309 Rud, Norway
}

\begin{abstract}
A new sensor based sorting system has been developed at Comex, based on complex image analysis. The sorting system employs different images generated by visible light and X-ray to provide maximal information about processed particles. Application of X-ray makes it possible to analyse the internal structure of the particles, which provides a $2 \mathrm{D}$ image with the valuable information. The advanced system for the texture and the pattern recognition is applied to analyse these images simultaneously, to provide the required identification of the particles for efficient separation. The paper describes a number of tests during sorting of $\mathrm{Cu}-\mathrm{Zn}$-Sn ores with high separation efficiency. As an example, the $\mathrm{Cu}$ content is enriched from $0.4 \%$ to $1.25 \%$ and the $\mathrm{Zn}$ content is increased from $0.83 \%$ to $2.24 \%$. In addition, the performance of the X-ray sorting system is described for enrichment of $\mathrm{Zn}-\mathrm{Pb}$ ore and compared with the heavy media separation technology. Simplifying the preconcentration through the use of the sorting technology can significantly reduce the cost of these operations. Finally, the operating data from the sorting system have been analysed regarding a potential use for controlling the plant operation. This can bring the new tools for control strategies for the complex processing plants.
\end{abstract}

\section{Current technology}

Processing of metal ores including zinc and lead, is mainly based on gravity separation equipment. It often includes applying dense media separation systems, especially for large particles. Such equipment provides an efficient separation, however, it has a number of disadvantages and limitations. The most important of them can are listed below:

- It is necessary to apply complicated media conditioning equipment to achieve a stable liquid density, like pumps, dosage systems, mixers, density sensors, etc. - It requires applying heavy density materials like ferrosilicon, which are expensive.

- The high density materials must be washed thoroughly from the particles to avoid loses and limit product contamination.

- The system requires use of water, which must be purified and recycled. It also requires heating in low temperature environments.

- The washing system is mechanically complicated with many moving parts, which require servicing and maintenance.

- The complete system requires a lot of space in form of both height and foot print area.

All these disadvantages provide a high cost of operation [1] especially for the low production capacity. It is very often not feasible to apply such systems for low or medium production scale. Consequently, it is necessary

\footnotetext{
* Corresponding author: jacek.kolacz@comex-group.com
}

to build the central processing plants, which require more transportation lines and complicated logistics.

Finally, the dense media separation process provides a relatively low separation sharpness. The particles, which have very high or very low density, when compared to the suspension density, will very quickly sink or float, thus providing a good separation effect. However, when the separated material has a density closer to the suspension, the sinking or floating effect will require more time and this can create more chances for misplaced particles during this process. Such effect is not taking place when sensor based sorting is applied.

\section{X-ray sorting technology}

Application of the sensor based sorting systems, without any doubt, brings a lot of possible advantages in many mineral operations. The main advantage of the new system is related to its universality and at the same time very sophisticated image processing functions, which can be carried out in the same processing unit [2]. Figure 1 shows the CXR-1000 system from Comex with $1 \mathrm{~m}$ belt width, in the configuration where many different analysed parameters can be used to provide particle separation. The image analysis system includes a camera installed either over the transport belt conveyor or at its discharge end. The system includes the X-ray attenuation analysis realized by the XRT system in the central part of the conveyor belt. The sorting system can be used with both 
optical and XRT analysis or separately depending on an application.

Particle recognition used to separate different materials is based on a complex shape and colour analysis where the particles can also be identified by over 20 parameters used for shape description. Some of them are: diameter in different orientations, perimeter, centre of mass, moment of inertia, particle elongation factor, edge sharpness, etc. Additional combinations of these parameters can also be used for distinguishing particles of interest. The surface of particles where different colours or contours vary in intensity and frequency can be analysed by FFT filtration (Fast Fourier Transformation) to recognize differences in texture and structure of the processed particles. This analysis brings much more complex information about the analysed particles rather than colour recognition alone. Finally, the XRT picture is integrated into the optical analysis, which provides a lot more information about the particle surface properties and its internal structure. All these sophisticated analysing functions require a lot of computation power and they have to be optimized to allow high capacity sorting. This is done by special program architecture and algorithm solutions allowing efficient management of the calculation routines and sorting priorities. This allows achieving still high separation capacity and extremely high efficiency [2], where the product purity can reach even $99.9 \%$. Such results can be achieved in different production scale depending on the process requirements [3].

The sorting system as shown above can be configured with different sensors depending on the particular application. In the test work as described in this paper, only the X-ray sensor was applied since the optical properties did not show any possibility for differentiation of particles of interests. The X-ray sensor provided possibility to calculate a density of separated particles. Therefore, it was possible to compare this new sorting technology with dense media separation as the traditional processing method in this process. Such sensor based sorting systems are already successfully tested and applied in the metal ore processing like iron ore [2] including $\mathrm{Zn} / \mathrm{Pb}$ ore [4] and coal [5].

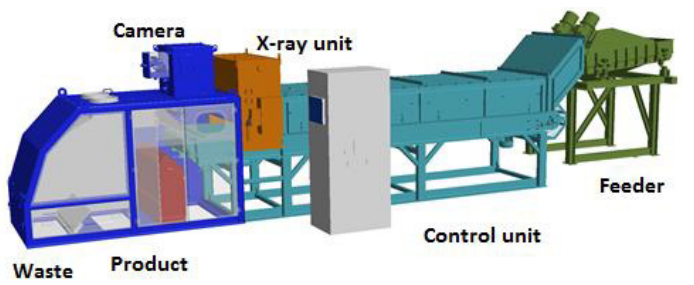

Fig. 1. Comex CXR sorting system - configuration with the Xray and the optical analysis.

Application of the sensor based sorting is very attractive when compared to other separation technologies. It provides the following advantages, when compared to the DMS systems:

- Possibility to apply in small capacity production lines

- Small space requirement

- No use of water (it is completely dry technology)
- No complicated additional equipment like, pumps, mixers, conditioners, recuperation devices, instrumentation, etc.

- No requirement of high density powder (ferrosilicon)

- Lower investment cost (about 25-50\%)

- Very low operating cost (about 15-20 time lower)

It was therefore very interesting to compare the separation efficiency using the similar input material.

\section{Comparison of the DMS and X-ray separation results}

The DMS and the X-ray sorting system have been compared on the base of the same feed material. The test work was performed in the zinc and lead mine DPM „Olkusz - Pomorzany” in Poland, where the DMS system was applied together with flotation as the main enrichment process [4]. The DMS system was employing the Wemco unit operating in $20-60 \mathrm{~mm}$ size range. It was possible to sample the input and outlet fractions and carry out the necessary chemical analysis and gravity separation tests. The samples included few hundred $\mathrm{kg}$ material fractions, taken under the stable operating conditions of the DMS system. The results of the detailed analysis of the feed, sink and float fractions are shown in Table 1.

Table 1. Separation results in the Wemco DMS system.

\begin{tabular}{|c|c|c|c|c|c|c|c|}
\hline \multirow{2}{*}{ Fraction } & \multirow{2}{*}{$\begin{array}{c}\text { Rec. } \\
{[\%]}\end{array}$} & \multicolumn{3}{|c|}{ Grade [\%] } & \multicolumn{3}{|c|}{ Recovery [\%] } \\
\cline { 3 - 8 } & & Zn & Pb & Fe & Zn & Pb & Fe \\
\hline Feed & 100 & 1.80 & 0.80 & 4.17 & 100 & 100 & 100 \\
\hline Sink (heavy) & 19.13 & 7.63 & 3.93 & 16.80 & 81.31 & 93.77 & 76.77 \\
\hline Float (light) & 80.87 & 0.41 & 0.06 & 1.20 & 18.69 & 6.23 & 23.23 \\
\hline
\end{tabular}

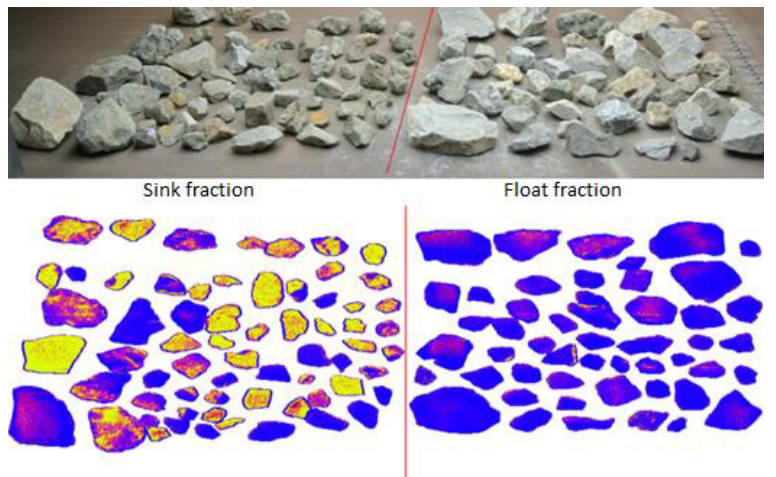

Fig. 2. Picture of the DMS outlet fractions together with the Xray image of the same particles.

In addition, the sink and float fractions, were analysed by the Comex CXR sorting unit to investigate the content of high and low density particles. Figure 2 shows these fractions in form of the optical picture and the X-ray image. The blue colure shows the low density particles and the red and yellow colours indicate the high density material. It can be noticed that the sink fraction still contains the low density particles which can be recovered by the sorting unit and separated to the waste fraction. It was therefore concluded that the separation process in the 
X-ray sorting unit might provide better result in form of increased recovery or grade values.

The Comex CXR-1000 X-ray sorting unit with $1 \mathrm{~m}$ belt width was used for testing. It was fed with the same material as the feed sample taken from the DMS unit. It was therefore possible to compare the separation effect from both separating units using the identical feed material composition. The separation results are shown in Table 2.

Table 2. Separation results in the CXR sorting system.

\begin{tabular}{|c|c|c|c|c|c|c|c|}
\hline \multirow{2}{*}{ Frac. } & \multirow{2}{*}{$\begin{array}{c}\text { Rec. } \\
{[\%]}\end{array}$} & \multicolumn{3}{|c|}{ Grade [\%] } & \multicolumn{3}{c|}{ Recovery [\%] } \\
\cline { 3 - 8 } & & $\mathbf{Z n}$ & $\mathbf{P b}$ & $\mathbf{F e}$ & $\mathbf{Z n}$ & $\mathbf{P b}$ & $\mathbf{F e}$ \\
\hline Feed & 100 & 1.80 & 0.80 & 4.17 & 100 & 100 & 100 \\
\hline High dens. & 10.96 & 5.42 & 15.36 & 79.16 & 87.12 & 53.40 & 10.96 \\
\hline Low dens. & 87.00 & 0.43 & 0.12 & 2.00 & 20.84 & 12.88 & 46.60 \\
\hline
\end{tabular}

When comparing the results in Table 1 and 2 , it can already be noticed that the a different enrichment level of investigated minerals was registered for various recoveries. For better illustration, the rade and the recovery points were shown on Figure 3.

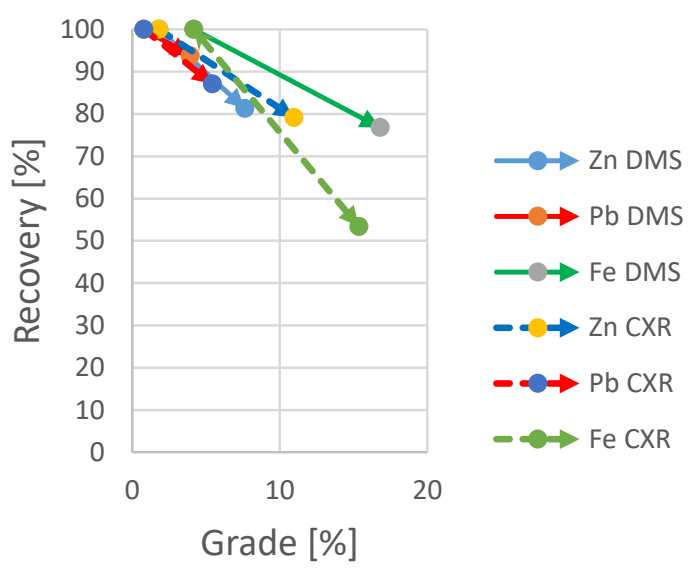

Fig. 3. Grade-recovery illustration for the DMS and X-ray sorting processes.

For the DMS process, the $\mathrm{Pb}$ content was with lower grade but higher recovery, when compared to the X-ray sorting. However, this can be adjusted by setting of the sorter to achieve a similar level of both parameters. Regarding the $\mathrm{Zn}$ separation, there is a significant difference in grade for the similar recovery level, where the X-ray sorting provides almost $11 \%$ grade, while DMS only $7.6 \%$, for the similar recovery of about $80 \%$. Finally, the recovery of $\mathrm{Fe}$ is much lower for and the grade is reduced, which is beneficial for this particular process of $\mathrm{Zn}-\mathrm{Pb}$ production.

\section{Other sorting applications}

Another application of the CXR sorting unit is shown for the $\mathrm{Cu}-\mathrm{Zn}-\mathrm{Sn}$ ore in one of the mines in China. In this case the ore was investigated in two main material streams where the $\mathrm{Cu}$ concentration was the main component in the first case, and the second stream contained mainly $\mathrm{Zn}$ and Sn. In this case the material was processed by the external processing plant where the large quantities of the ore had to be transported with significant distances. The sorting solution with the CXR system was suggested to provide improvements in the overall process. The separation results are shown in Table 3 for the $\mathrm{Cu}$ ore. In this case, the feed material containing $0.404 \%$ of $\mathrm{Cu}$ was enriched to $1.258 \%$ with $85.65 \%$ recovery of $\mathrm{Cu}$. At the same time the concentrate stream after sorting system, corresponded to only $27.52 \%$ of the incoming mass. It means the pre-concentrated material was significantly reduced in quantity before further processing.

Table 3. $\mathrm{Cu}$ ore separated in the CXR sorting system.

\begin{tabular}{|l|c|c|c|c|c|}
\hline \multirow{2}{*}{ Fraction } & \multirow{2}{*}{$\begin{array}{c}\text { Yield } \\
{[\%]}\end{array}$} & Density & \multicolumn{3}{|c|}{ Test Elements [\%] } \\
\cline { 4 - 6 } & & & $\mathbf{C u}$ & $\mathbf{Z n}$ & Sn \\
\hline Cu Conc. & 27.52 & 3.09 & 1.258 & 0.989 & 0.299 \\
\hline Cu Tailing & 72.48 & 1.92 & 0.080 & 0.151 & 0.096 \\
\hline Cu Feed & 100 & 2.24 & 0.404 & 0.382 & 0.152 \\
\hline \multirow{2}{*}{ Fraction } & $\begin{array}{c}\text { Yield } \\
{[\%]}\end{array}$ & $\begin{array}{c}\text { Density } \\
{[\mathbf{k g} / \mathbf{I}]}\end{array}$ & \multicolumn{4}{|c|}{ Recovery [\%] } \\
\cline { 4 - 7 } & & $\mathbf{C u}$ & $\mathbf{Z n}$ & Sn \\
\hline Cu Conc. & 27.52 & 3.09 & 85.65 & 71.32 & 54.18 \\
\hline Cu Tailing & 72.48 & 1.92 & 14.35 & 28.68 & 45.82 \\
\hline Cu Feed & 100 & 2.24 & 100 & 100 & 100 \\
\hline
\end{tabular}

Table 4. $\mathrm{Zn} / \mathrm{Sn}$ ore separation in the CXR sorting system.

\begin{tabular}{|c|c|c|c|c|c|}
\hline \multirow{2}{*}{ Description } & \multirow{2}{*}{$\begin{array}{c}\text { Yield } \\
{[\%]}\end{array}$} & \multirow{2}{*}{$\begin{array}{c}\text { Density } \\
{[\mathbf{k g} / \mathbf{l}]}\end{array}$} & \multicolumn{3}{|c|}{ Test Elements [\%] } \\
\cline { 4 - 6 } & {$[\% \mathbf{C u}$} & $\mathbf{Z n}$ & $\mathbf{S n}$ \\
\hline Zn/Sn Conc. & 28.30 & 2.87 & 0.145 & 2.249 & 0.655 \\
\hline $\begin{array}{c}\text { Zn/Sn } \\
\text { Tailing }\end{array}$ & 71.70 & 2.65 & 0.023 & 0.273 & 0.097 \\
\hline Zn/Sn Feed & 100 & 2.71 & 0.058 & 0.832 & 0.255 \\
\hline Description & $\begin{array}{c}\text { Yield } \\
{[\%]}\end{array}$ & $\begin{array}{c}\text { Density } \\
{[\mathbf{k g} / \mathbf{l}]}\end{array}$ & \multicolumn{4}{|c|}{ Recovery [\%] } \\
\cline { 4 - 6 } & $\mathbf{C u}$ & $\mathbf{Z n}$ & $\mathbf{S n}$ \\
\hline Zn/Sn Conc. & 28.30 & 2.87 & 71.33 & 76.48 & 72.72 \\
\hline $\begin{array}{c}\text { Zn/Sn } \\
\text { Tailing }\end{array}$ & 71.70 & 2.65 & 28.67 & 23.52 & 27.28 \\
\hline Zn/Sn Feed & 100 & 2.71 & 100 & 100 & 100 \\
\hline
\end{tabular}

The similar results were achieved for the $\mathrm{Zn}-\mathrm{Sn}$ ore as shown in Table 4 . In this case, the feed contained $0.832 \%$ of $\mathrm{Zn}$ and $0.255 \%$ of Sn. After sorting, the metal content was increased to $2.249 \%$ of $\mathrm{Zn}$ and $0.655 \%$ of Sn with the recoveries of $76.48 \%$ and $72.72 \%$ correspondingly. At the same time, the material quantity was reduced to $28.3 \%$ of the total incoming mass to the sorting process. Again, this provided huge savings in terms of the further processing cost.

\section{Operating cost improvements}

Sorting systems have the important limitation related to the capacity of the single processing unit. This is related to the operating principle where the sorting process is carried out in the mono-layer of the particles introduced to the sorting unit. It means that higher throughputs must 
be provided by the parallel configuration of the sorting units. Dry X-ray separation equipment provided by Comex fulfils this main cost requirement for multiple stage separation keeping the total cost at a competitive level. The resent development and progress in microelectronics provided quite a number of new possible solutions within automation and separation techniques. Comparison with the traditional processing methods like DMS, indicate very clearly that the dry X-ray sorting systems can be very competitive regarding investment cost, operating cost and other environmental aspects like no need of water. Simple comparison of the DMS washing plant and the sorting plant based on CXR Comex sorters, is shown in Table 5.

Table 5. Cost comparison between the traditional washing plant and the Comex dry beneficiation plant.

\begin{tabular}{|c|c|c|c|}
\hline Option & & $\begin{array}{c}\text { DMS } \\
\text { plant }\end{array}$ & $\begin{array}{c}\text { Comex dry } \\
\text { sorting plant }\end{array}$ \\
\hline $\begin{array}{c}\text { Processing } \\
\text { capacity }\end{array}$ & {$[\mathrm{MTPA}]$} & $4.5-5$ & $4.5-5$ \\
\hline $\begin{array}{c}\text { Water } \\
\text { consumption }\end{array}$ & {$[\mathrm{l} / \mathrm{t}]$} & $1-2$ & 0.01 \\
\hline Investment cost & {$[\mathrm{USD}]$} & $15 \mathrm{mln}$ & $10-12 \mathrm{mln}$ \\
\hline Operating cost & {$[\mathrm{USD} / \mathrm{t}]$} & $\mathbf{1 - 1 . 5}$ & $\mathbf{0 . 0 5 - 0 . 1}$ \\
\hline
\end{tabular}

The traditional DMS plant is characterised by a simple operating principle, however, it requires a lot of moving parts, motors, belts, pumps, magnetic recuperation as well as access to water and heavy media like ground magnetite or ferrosilicon. This result in high both investment and especially operating cost. Based on the average plant size providing 5 MTPA the investment cost is about $15 \mathrm{mln}$ USD and the operating cost ranges about 1-1.5 USD per ton. In addition there is a cost related to water supply which is very difficult to calculate especially for hot climate countries.

For the alternative process where the CXR sorters are employed, the separation can be done in combination of parallel-serial connections of the sorting units and the necessary screening equipment. The investment cost ranges between 10-12 mln USD and the operating cost is about 0.05-0.1 USD per ton. It means the operating cost of the X-ray sorting plant is about 15-20 times lower then the corresponding process employing DMS. The benefit of using the dry X-ray sorting plant is growing rapidly when the processing capacity is lower. For smaller mines, it is often not feasible to build the washing plant while the dry sorting system having smaller capacity, can easily be applied.

\section{Data processing potential}

The sorting system is usually installed at the high throughput streams, which are at the early stage of processing. It means many particles are passing the sensors of the sorting equipment and these particles very often correspond to the ROM material or the main material introduced for the enrichment process. By collecting this data on-line, it is possible to control the rest of the process by adjusting its parameters to obtain the optimal conditions and adjusting the flowsheets streams. The control system can adjust e.g. the reagents concentration used in floatation based on the information about the metal or rejects concentration in the ore. It is also possible to use a fuzzy logic control to adjust the processing paths in the flowsheet to get the best performance of the plant. It brings new possibilities for optimising the complex processing plants. This knowledge has never been used before and it will be further investigated in the future work.

\section{Conclusions}

The CXR X-ray sorting unit can provide a similar or even better separation effect than DMS system, during preconcentration of the $\mathrm{Zn}-\mathrm{Pb}$ ore before further processing. The similar separation process can be realised by X-ray sorting as a much simpler system, regarding lower number of moving parts, lower complication level, as well as reduced maintenance and servicing. No need of water and the high density weight material, can be important for more flexible and more environmentally friendly operation. Finally, in terms of the reduced investment and operating cost (by 15-20 times), it can dramatically improve the mine operation regarding economic aspects. Finally, the sorting results can be transferred to the valuable data describing the input material. Such information can be used for controlling the rest of the processing plant by adjusting the control models for flotation regents and different flowsheet configurations.

\section{References}

1. Wills B.A., Wills' Mineral Processing Technology: An Introduction to the Practical Aspects of Ore Treatment and Mineral Recovery (2006)

2. Kolacz J. Sensor based sorting with signal pattern recognition: the new powerful tool in mineral processing (XXVII International Mineral Processing Congress, Santiago-Chile,106-115, 2014)

3. Kolacz J. Efficient scale up of sensor based sorting systems. XXVIII International Mineral Processing Congress, Quebec, Canada, 2016)

4. Wieniewski at al., A. Wieniewski, E. Szczerba, A. Nad, R. Luczak, J. Kolacz, Szewczuk, Evaluation of the application possibilities of modern separation techniques for pre-concentration of the $\mathrm{Zn}-\mathrm{Pb}$ ore, (XI International Non-Ferrous Metals Conference ICNOP, Trzebieszowice, Poland, 2015)

5. Kolacz J. Dry coal beneficiation by sensor based sorting (Mineral Engineering Conference, Szczawnica, Poland, 2015) 